\title{
POLARIZATION REMOTE SENSING PHYSICAL MECHANISM, KEY METHODS AND APPLICATION
}

\author{
Bin Yang ${ }^{\mathrm{a}}$, Taixia $\mathrm{Wu}^{\mathrm{b}}$,Wei Chen ${ }^{\mathrm{c}}$, Yanfei Lic ${ }^{\mathrm{c}}$, Juri Knjazihhin ${ }^{\mathrm{d}}$, Anand Asundie Lei Yan ${ }^{\mathrm{a}, *}$ \\ (a. Institute of Remote Sensing and Geographic Information, School of Earth and Space Sciences, Peking University, Beijing \\ 100871, China; \\ b. College of Earth Science and Engineering, Hohai University, Nanjing 210098, China;
}

c. School of Earth Sciences and Surveying and Mapping Engineering, China University of Mining and Technology (Beijing),

Beijing 100083, China;

d.Boston University, Boston MA-02215, USA;

e.Nanyang Technological University, Singapore 639798)

(* Corresponding author: Yan Lei, E-mail: 1yan@pku.edu.cn)

KEY WORDS: Polarized remote sensing; Polarization pattern of the skylight; Atmospheric polarization neutral point; Polarization device; Calibration system

\begin{abstract}
:
China's long-term planning major projects "high-resolution earth observation system" has been invested nearly 100 billion and the satellites will reach 100 to 2020 . As to $2 / 3$ of China's area covered by mountains, it has a higher demand for remote sensing. In addition to light intensity, frequency, phase, polarization is also the main physical characteristics of remote sensing electromagnetic waves. Polarization is an important component of the reflected information from the surface and the atmospheric information, and the polarization effect of the ground object reflection is the basis of the observation of polarization remote sensing. Therefore, the effect of eliminating the polarization effect is very important for remote sensing applications.

The main innovations of this paper is as follows: (1) Remote sensing observation method. It is theoretically deduced and verified that the polarization can weaken the light in the strong light region, and then provide the polarization effective information. In turn, the polarization in the low light region can strengthen the weak light, the same can be obtained polarization effective information. (2) Polarization effect of vegetation. By analyzing the structure characteristics of vegetation, polarization information is obtained, then the vegetation structure information directly affects the absorption of biochemical components of leaves. (3) Atmospheric polarization neutral point observation method. It is proved to be effective to achieve the ground-gas separation, which can achieve the effect of eliminating the atmospheric polarization effect and enhancing the polarization effect of the object.
\end{abstract}

\section{Introduction}

Polarization is the four main physical characteristics of remote sensing electromagnetic waves with light intensity, frequency and phase. Polarization is an important part of the reflected information of surface and atmospheric system. It is an important for inversion of surface and atmospheric information in the field of remote sensing. At present, the research of optical remote sensing is focused on remote sensing, but the research on polarized remote sensing is less, and most of them are ignored or removed as noise. Vegetation is one of the typical features, Based on this, analyzing the structure characteristics of vegetation, polarization information is obtained, and then the influence of polarization effect is verified.

\section{Polarization of light}

The asymmetry of the direction of vibration of the wave is called polarization, which is the unique phenomenon of shear wave. Polarization (called polarization in the microwave spectrum) is an important feature of electromagnetic waves. 
Earth's surface and atmospheric targets in the reflection, scattering and transmission and emission of electromagnetic radiation in the process, they will produce their own nature of the characteristics of the polarization, that is, the polarization characteristics of the target contains a variety of information. Stokes parameters can be expressed as I, Q, U, V, ie

$$
\left\{\begin{array}{c}
I=I_{0^{\circ}}+I_{90^{\circ}} \\
Q=I_{90^{\circ}}-I_{0^{\circ}} \\
U=I_{+45^{\circ}}-I_{-45^{\circ}} \\
V=I_{r}-I_{l}
\end{array}\right.
$$

Where $\mathrm{I}$ is the light intensity, Q, U represent the linearly polarized light intensity in both directions, $\mathrm{V}$ represents the circularly polarized light intensity, I0 is the total intensity of the light, $I_{0^{\circ}}, I_{90^{\circ}}, I_{+45^{\circ}}, I_{-45^{\circ}}$ represent the linearly polarized light in the $0^{\circ}, 90^{\circ},+45^{\circ},-45^{\circ}$ direction of the ideal polarizer placed on the optical propagation path. $I_{r}$ and $I_{l}$ represent the intensity of the left and right of the circularly polarized light.

The degree of polarization of the incident light is

$$
\mathrm{P}=\frac{\sqrt{Q^{2}+U^{2}+V^{2}}}{I}
$$

The polarization azimuth of the incident light is

$$
\varphi=\frac{1}{2} \tan ^{-1}\left(\frac{U}{Q}\right)
$$

\section{Polarization effect}

At present, the conventional remote sensing can only get good images in the case of moderate $1 / 3$ light, and the rest is too bright (such as solar storms, stellar objects or water flares), and too dark (such as major natural disasters or remote very weak light planetary detection) is difficult to obtain, which has become the advanced space exploration and surface geological disasters such as remote sensing observation of the bottleneck. Polarization based on the observed physical and chemical properties of different physical and chemical properties of a strong contrast ratio, that is, strong light "weakened", low light "enhanced" physical mechanism, to solve this problem to provide new possibilities.

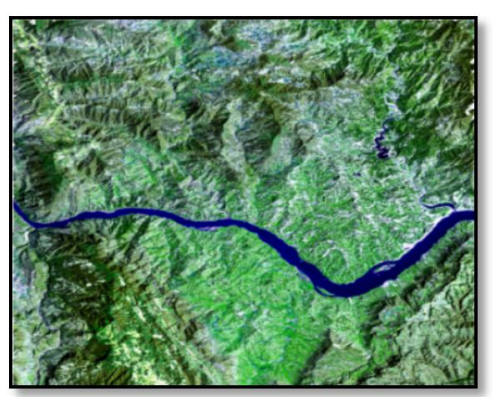

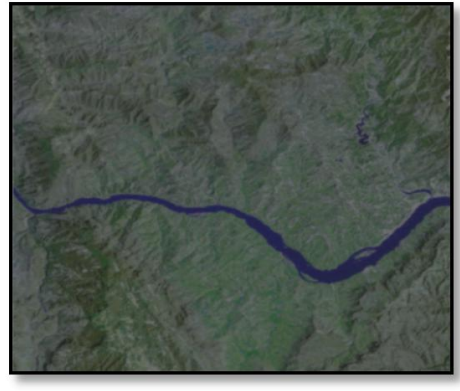

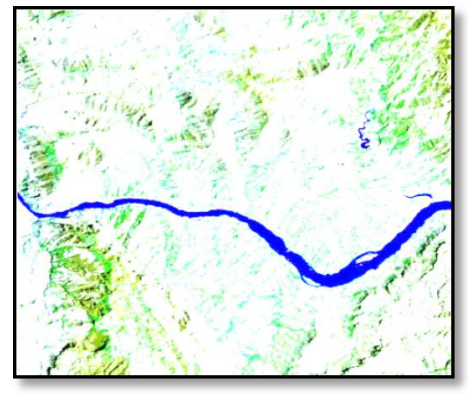

Figure 1. Remote image of different intensity (clear surface, too dark and black, too bright and saturated )

Assuming that the incident radiation is the unit radiation, the radiation will have multiple scattering between the surface, the surface and both, and the final radiation obtained by the sensor is the sum of the three. In general, the radiation incident to the surface, or is directly scattered back into the atmosphere, or absorbed, or into the surface of the internal. Into the surface of the radiation inside the surface with the material, such as moisture and other biochemical effects, will be part of the scattering, part of the absorption. However, unlike surface scattering, this part of the scattering is related to the material content inside the surface, and its modulation of the radiation is reflected in the reflectivity, which is non-polarized (Knyazikhin Y, 2013a) (Knyazikhin Y, 2013b)in Figure 2.

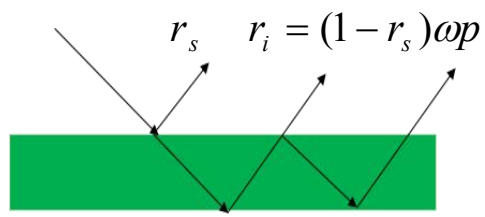

Figure 2. Schematic diagram of the interaction between radiation and surface

The probability of scattering is as follows: the probability that the surface is directly reflected is the probability of scattering upwards inside the surface, and the probability of scattering down is. According to the Fresnel principle, we know that the 
radiation directly reflected on the surface is partially polarized, that is to say, it can be further decomposed into:

$$
r_{s}=r_{s p}+r_{s n p}
$$

Which represents a linearly polarized portion and represents a portion.

Into the interior of the surface, with its interaction, the radiation is the total probability of scattering:

$$
r_{i}=\left(1-r_{s}\right) \omega \rho
$$

The probability of radiation being absorbed

$$
a_{i}=\left(1-r_{s}\right)(1-\omega)
$$

The probability of transmission downwards

$$
t_{i}=\left(1-r_{s}\right) \omega \tau
$$

The total energy of the radiation received by the sensor interacting with the surface and the interior can be simply expressed as:

$$
r=r_{s}+\left(1-r_{s}\right) \omega \rho
$$

The degree of polarization detected by the sensor is:

$$
p=\frac{r_{s p}}{r_{s}+\left(1-r_{s}\right) \omega \rho}
$$

In general, RSP can be considered constant, that is, regardless of the amount of wavelength. When the reflectivity of the surface is low, that is, the performance of the smaller value, from the visual effect, the target will appear very dark, then the degree of polarization $\mathrm{p}$ is a large value, then the realization of the "weak light" process; When the reflectivity of the surface is large, that is, the performance of the larger value, from the visual effect, the target will appear very bright, the degree of polarization $\mathrm{p}$ is shown as a small value, then the "light weakening" process.

\section{Polarization effect of canopy structure}

Leaf scattering spectrum is the key optical variable that conveys information about leaf absorbing constituents from remote sensing. It cannot be directly measured from space because the radiation scattered from leaves is affected by the 3D canopy structure(Knyazikhin et al., 2013a; Knyazikhin et al., 2013b; Knyazikhin et al., 2013c). In addition, some radiation is specular reflected at the surface of leaves. This portion of reflected radiation is partly polarized, does not interact with pigments inside the leaf and therefore contains no information about its interior. Very little empirical data are available on the spectral and angular scattering properties of leaf surfaces. Whereas canopy-structure effects are well understood, the impact of the leaf surface reflectance on estimation of leaf absorption spectra remains uncertain. We thus present empirical and theoretical analyses of angular, spectral, and polarimetric measurements of light reflected by needles and shoots of Pinus koraiensis and Picea koraiensis species. Figure 3 illustrates our samples.

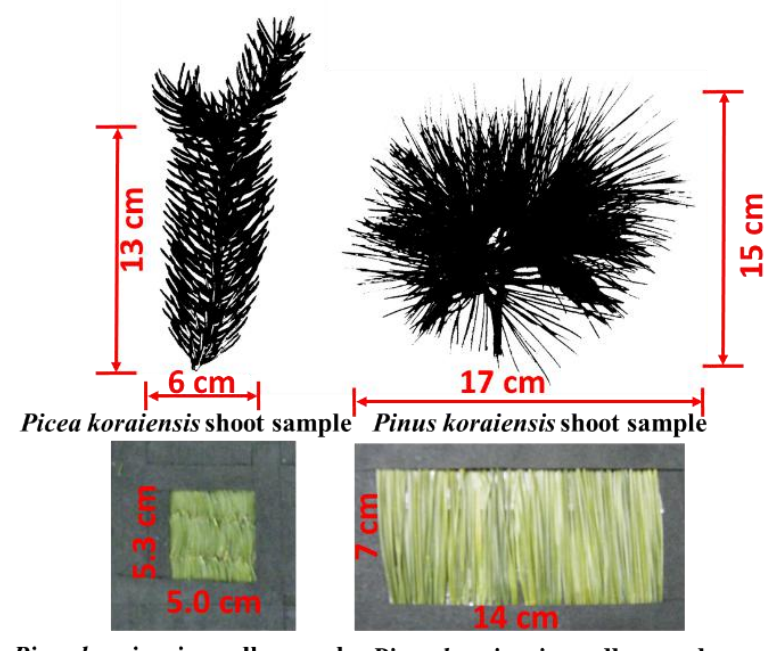

Picea koraiensis needle sample Pinus koraiensis needle sample

Figure 3. Samples of shoots and needles in the holder window. Sizes of the shoots were $13 \mathrm{~cm}$ by $6 \mathrm{~cm}$ (Picea koraiensis) and $15 \mathrm{~cm}$ by $17 \mathrm{~cm}$ (Pinus koraiensis). Dimensions of the holder windows were $5.3 \mathrm{~cm}$ by $5.0 \mathrm{~cm}$ (Picea koraiensis) and $7 \mathrm{~cm}$ by $14 \mathrm{~cm}$ (Pinus koraiensis).

The total radiation reflected by a leaf includes two components, specular and diffuse(Vanderbilt and Grant, 1985). The first component emanating from light reflected at the air-cuticle interface is polarized. The diffuse component results from photon interactions within the leaf and any large particle on the leaf surface. This portion of reflected radiation is not polarized. Polarization measurements help us to extract linearly polarization portion from the total radiation registered by the sensor. Radiation specular reflected from the needle sample surfaces exhibit weak spectral dependency, as expected from theory. It increases from negligible values in backscattering directions to about $17 \%$ in forward scattering directions. The shoot sample polarized directional-conical reflectance factor (PDCRF) shows a similar behavior(Yang et al., 2016). Its magnitude, however, is reduced by a factor of about 10 , as Figure 4 shows. 


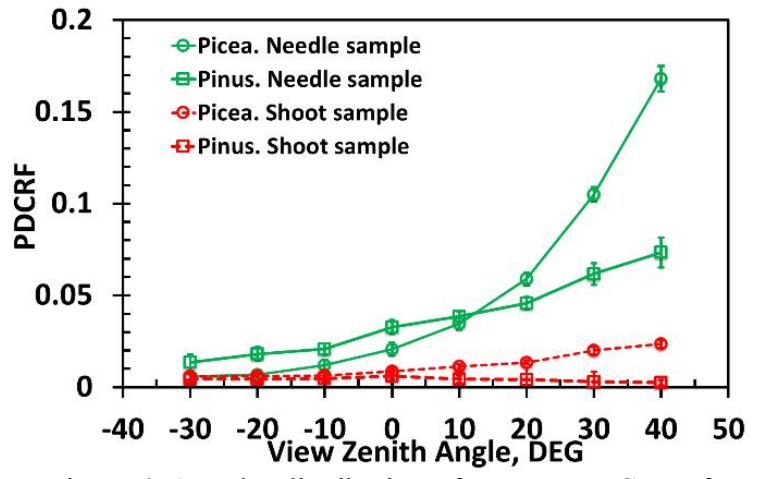

Figure 4. Angular distribution of average PDCRF of needle samples (solid lines) and shoot samples (dashed lines) averaged over 450-950 nm. Vertical bars denote \pm 1 standard deviation.

Ignoring polarization portion of reflected radiation, however, can cause an overestimation of the scattering coefficient (Figure 3). The impact decreases from strongly $(17 \%-140 \%, 450-500 \mathrm{~nm})$ to weakly $(<4 \%, 800-950 \mathrm{~nm})$ absorbing wavelengths (Figure 5). Note that our approach provides a lower bound on the impact, i.e., a "true" impact is stronger(Yang et al., 2016).

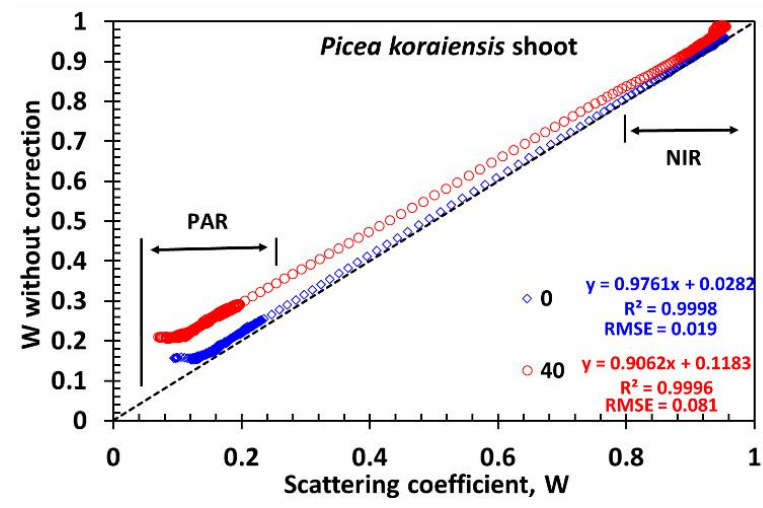

Figure 5. Correlation between scattering coefficients of the Picea koraiensis shoot derived with (horizontal axis) and without (vertical axis) correction for the needle surface effects. In this example, relative differences are $17 \%-140 \%$ in blue $(450-500 \mathrm{~nm}), \quad 3 \%-74 \%$ red $(600-650 \mathrm{~nm})$, $3 \%-59 \%$ green $(520-580 \mathrm{~nm})$ and below $4 \%$ in the near infrared (800-950 nm) spectral intervals.

To summarize, the angular, spectral and polarimetric data convey information about properties of the needle surfaces, shoot structural organizations and needle optics. This information is required to retrieve the needle albedo, which is directly related to the absorption spectra of leaf biochemical constituents.

\section{Ground - gas separation of new atmospheric windows}

Atmospheric decay is the largest source of error in Earth observation, with an effect of up to $5-30 \%$.
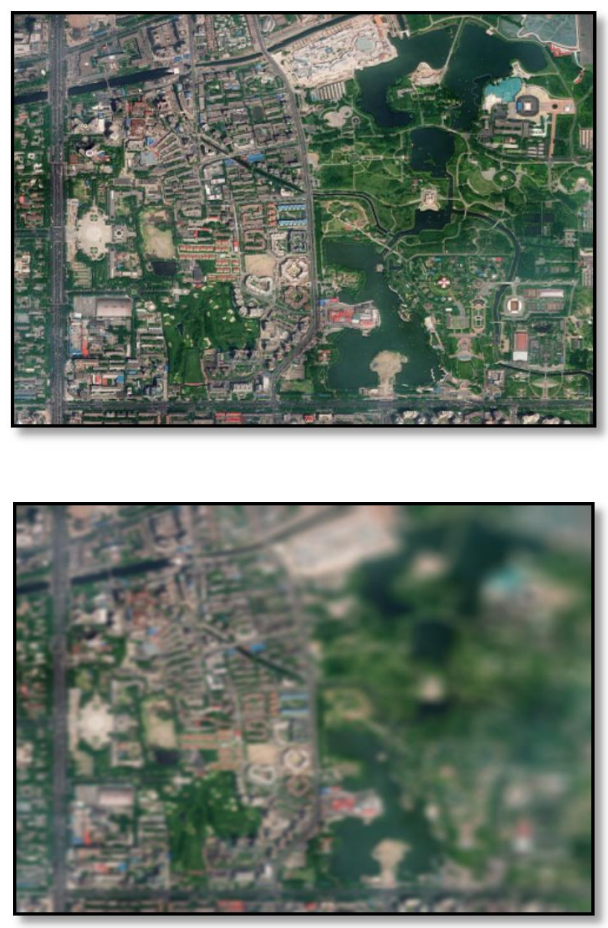

Figure 6. Remote sensing images with small atmospheric impacts (left) and large atmospheric impacts (right)

In order to verify the theoretical feasibility of using the neutral point for the separation of ground-air polarization effects(Lee, 1998), a ground verification experiment was designed. The experiment is to polarize the same region at the neutral and non-neutral locations, respectively, and then compare the polarized parameters of the imaging. Figure 7 shows the geometric schematic of the observations for ground experiments, (a) for observations at neutral point, and (b) for observing at non-neutral locations. The degree of polarization on the connection between the surface and the neutral point is zero. The imaging device used in the experiment is Nikon D200 SLR digital camera, placed in front of the camera iodine polarizer, change the polarizer axis and the reference axis angle, respectively, $0^{\circ}, 60^{\circ}, 120^{\circ}$ three angles of the data.

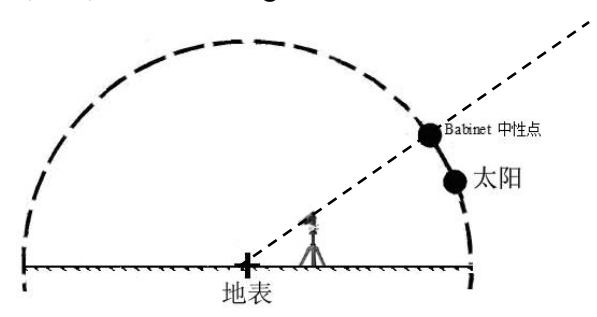




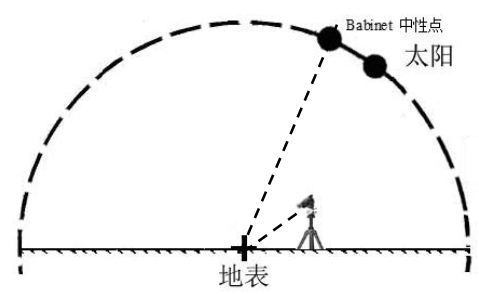

(a) Neutral observation (morning) (b) Non-neutral observation (noon)

Figure 7. Geometric schematic diagram of ground experiment validation

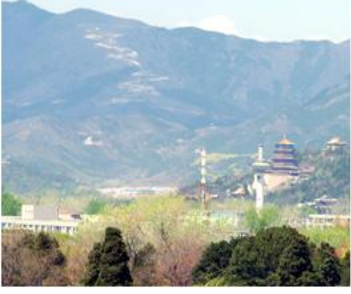

Non - neutral observation

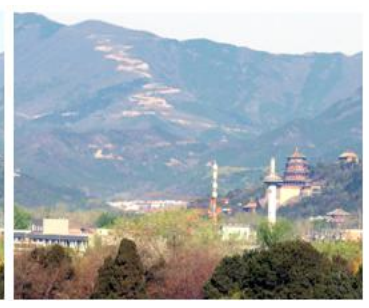

Neutral observation unpolarized images

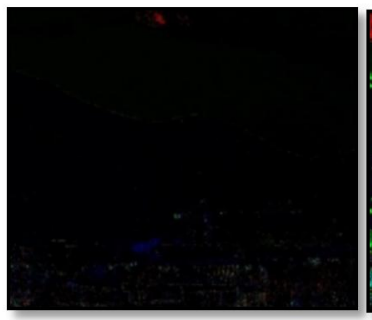

Non - neutral observation

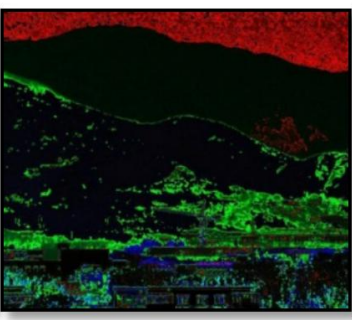

Neutral observation (b) polarized images

Figure 8. Comparison of polarization parameters for neutral point imaging and non-neutral point imaging

(Beijing Summer Palace)

Figure 8 shows the polarization parameters for the neutral point imaging and the non-neutral point imaging. The left image is a non-neutral view of the image, the right image is a neutral observation of the image.

Figure 8. (a) images with no polarizing film, the visibility of the atmosphere is high, both in the neutral area observation or in the non-neutral area observation, the image is relatively clear; neutral observation of the image (right) Reflected on the terrain information than the non-neutral observation of the image (right) to reflect the information to be rich.

The polarimetric images of Fig. 8 (b) need to be studied, because the degree of polarization reflects the physical quantity of the magnitude of the degree of polarization of the object, and the image, which is difficult to separate from the ground-gas effect mentioned above, refers to the polarimetric image. At the neutral point of observation of the degree of polarization image (right), regardless of the Summer Palace Wanshou Mountain Buddha Xiang Court (distance from the shooting point of about $3.1 \mathrm{~km}$ ) or the distance of the Western Hills (distance from the shooting point of about 6-8 $\mathrm{km}$ ) are clearly visible, especially the mountain bare soil (or road) information and images have a good fit. And in the non-neutral point of view of the polarization image (left), near the ground information has a good performance, but the distant mountain information is much weaker, only faint Show the outline of the mountain. This means that the polarized information of the distant mountain cannot be obtained when observing non-neutral points. It also shows that as the distance from the shooting point to the feature increases, the polarization effect of the atmosphere increases, and the polarization information of the feature becomes weak. Comparing the four images of Figure. 8, the amount of information on the polarimetric image (right) of the neutral point observations is much larger than the amount of information on the non-neutral point of the polarimetric image (left), especially for the far Distance of the target. This shows that the imaging of the neutral region can achieve the effect of eliminating the atmospheric polarization effect and enhancing the polarization effect of the object. The feasibility of using the neutral point for ground - gas separation method is proved experimentally.

\section{Conclusions}

Conventional remote sensing can only get good images in $1 / 3$ light conditions, and the rest is too bright (such as solar storms, stellar celestial bodies or water flares), too dark (such as major natural disasters or remote very weak light planetary detection), Which became the advanced space exploration and surface geological disasters such as remote sensing observation of the bottleneck. Polarization based on the observed physical and chemical properties of different physical and chemical properties of a strong contrast ratio, that is, light "weakened", low light "enhanced" physical mechanism, to solve this problem to provide new possibilities. Neutral point regional imaging can achieve the effect of eliminating the atmospheric polarization effect and enhancing the polarization effect of the object.

\section{References:}

Knyazikhin, Y., Lewis, P., Disney, M.I., Mõttus, M., 
Rautiainen, M., Stenberg, P., Kaufmann, R.K., Marshak, A., Schull, M.A., Carmona, P.L., 2013a. Reply to Ollinger et al.: Remote sensing of leaf nitrogen and emergent ecosystem properties. Proceedings of the National Academy of Sciences of the United States of America 110, E2438-E2438.

Knyazikhin, Y., Lewis, P., Disney, M.I., Stenberg, P., Mõttus, M., Rautiainen, M., Kaufmann, R.K., Marshak, A., Schull, M.A., Latorre, C.P., 2013b. Reply to Townsend et al.: Decoupling contributions from canopy structure and leaf optics is critical for remote sensing leaf biochemistry. Proceedings of the National Academy of Sciences of the United States of America 110, E1075-E1075.

Knyazikhin, Y., Schull, M.A., Stenberg, P., Mõttus, M., Rautiainen, M., Yang, Y., Marshak, A., Latorre, C.P.,
Kaufmann, R.K., Lewis, P., 2013c. From the Cover: PNAS Plus: Hyperspectral remote sensing of foliar nitrogen content. Proceedings of the National Academy of Sciences of the United States of America 110, 185-192.

Lee, R.L., 1998. Digital imaging of clear-sky polarization. Applied Optics 37, 1465.

Vanderbilt, V.C., Grant, L., 1985. Plant Canopy Specular Reflectance Model. IEEE Transactions on Geoscience \& Remote Sensing GE-23, 722-730.

Yang, B., Knyazikhin, Y., Lin, Y., Yan, K., Chen, C., Park, T., Choi, S., Mõttus, M., Rautiainen, M., Myneni, R., 2016. Analyses of Impact of Needle Surface Properties on Estimation of Needle Absorption Spectrum: Case Study with Coniferous Needle and Shoot Samples. Remote Sensing 8. 